\title{
FAT TISSUE AND SYNTHESIZED BY HER ADIPOKINES AS MARKERS INDICATING THE DEVELOPMENT AND PROGRESS OF OSTEOARTHRITIS
}

DOI: 10.36740/WLek202009102

\author{
Jaromir Jarecki ${ }^{1}$, Agnieszka Tomczyk-Warunek ${ }^{1}$, Mateusz Wicha' ${ }^{1}$, Anna Horecka ${ }^{2}$, Rudolf Blicharski ${ }^{1}$, \\ Maciej Dobrzyński ${ }^{3}$ \\ 'CHAIR AND DEPARTMENT OF REHABILITATION AND ORTHOPAEDICS, MEDICAL UNIVERSITY OF LUBLIN, LUBLIN, POLAND \\ ${ }^{2}$ CHAIR AND DEPARTMENT OF MEDICAL CHEMISTRY, MEDICAL UNIVERSITY OF LUBLIN, LUBLIN, POLAND \\ ${ }^{3}$ DEPARTMENT OF CONSERVATIVE DENTISTRY AND PEDODONTICS, WROCLAW, POLAND
}

\begin{abstract}
Arthrosis as osteoarthritis is a global problem that affects more and more people and is associated with severe chronic pain, reduced mobility and, consequently, disability. The etiology of degenerative disease is complex and depends on many factors. However, its course was not fully understood. One of the factors affecting the development of arthrosis is obesity. Obesity is a growing problem. Over the past 30 years, the number of overweight people has almost doubled. In people suffering from obesity, whose body mass index is above $30 \mathrm{~kg} / \mathrm{m}^{2}$, the risk of developing degenerative changes in articular cartilage is six times higher than the risk of developing this disease in people with normal body weight. Osteoarthritis is detected when the symptoms get worse where the changes are already at some stage. Therefore, a lot of research is currently underway to find suitable biomarkers, which would indicate the potential development of degenerative changes in the future and at the same time the possibility of inhibiting their activity. One of them may be adipokines, which are synthesized by adipose tissue and affect cartilage. In obese people, adipokines may contribute to the inflammation of the low charterer, which accompanies both obesity and arthrosis. These compounds can be specific biomarkers to assess the degree of progression and severity of osteoarthritis.

The aim: To assess the importance of obesity and adipokines produced by adipose tissue as specific markers of arthrosis.
\end{abstract}

KEY WORDS: osteoarthritis, obesity, biomarkers, adipokines

Wiad Lek. 2020;73(9 p. I):1818-1823

\section{INTRODUCTION}

Arthrosis is a degenerative disease of joints, which disturbs their function. It is created because of disturbances in the balance between synthesis and degradation of cartilage tissue. Joint cartilage is responsible for resorption of mechanical forces affecting the joints and allows for proper functioning of the joints, which involves the movement of articular surfaces against each other [1]. Changes in these processes lead to the appearance of pathologies, which lead to degeneration of hyaline cartilage. Because of that, a new bone tissue is formed, and the existing bone tissue's density is increased, which leads to hardening of the subchondral layer and finally leads to creating osteophytes in the edge of the articular surfaces, which are bone processes [2]. Also, because of these changes, changes in structure of joint capsule, muscles, ligaments and tendons [1].

Progressive changes in cartilage lead to the appearance of pain, which leads to lowering the physical activity of the person [2]. This sickness is one of the main factors leading to disability in developed countries. With aging of the society there is a growing number of patients affected by the disease. [3]. Nowadays arthrosis affects over $37 \%$ of people over the age of 60 and almost $80 \%$ of patients with the age over 65 [4-6].

In the literature however there are no clear indications regarding the actual number of patients affected by this disease, which can be caused by diversification of the examined groups of patients, differing in: the size of the experimental group, age, race etc. [7]. There are also differences between acquired results during the radiological examination, which is proven by the appearance of degenerative changes in the joint and the symptoms in patients. These are proven by epidemiological studies, which state, that in $40 \%$ of the patients with moderate changes in the hip joint and $60 \%$ with significant changes in knee joint suffer from arthrosis [7]. It is also important, that the disease happens more frequently in women than men, which is caused by menopause in women [7].

The costs of arthrosis are high, because the treatment against arthrosis is very costly, correct rehabilitation must be applied, and it also causes patients affected by the disease to be less active, at the same time lowering their ability to work. The osteoarthritis usually affects the knee joints, causing intense pain, lowering the quality of life. The disease also affects different joints, like MCP (meta- 
carpophalangeal joints), PIP (proximal interphalangeal joints) and DIP (distal interphalangeal joints) which are not categorized as joints which are affected by high mechanical loads, but also have a very important role [8]. The osteoarthritis also touches joints of the spine and feet [7].

The etiology of the disease is complicated, because it can be caused by many factors. They can be generally categorized into 2 categories: genetical and non-genetical, so environmental [9]. One of the factors causing arthrosis is obesity [10].

Obesity is confirmed, if the BMI (body mass index), which is the body mass coefficient of the patient is more than $30 \mathrm{~kg} / \mathrm{m}^{2}$. Obesity is becoming a real threat because of its increasing popularity [10]. Information from 2008 state, that over 1,4 billion adult people suffer from obesity, with more than 300 million men and 200 million women are morbidly obese, with BMI over $40 \mathrm{~kg} / \mathrm{m}^{2}$. The number of people suffering from obesity increases daily. In the last 30 years the number of obese people increased over 2 times. Too high BMI increases the risk of many diseases to appear, involving the osteoarthritis [11].

In literature there are many information stating that in patients with high BMI (higher than $30 \mathrm{~kg} / \mathrm{m}^{2}$ ) the risk of osteoarthritis to appear is six times greater than in patients with correct body mass [11].

Arthrosis is just one result of obesity which appears in the hip and knee joints. Available research shows, that osteoarthrosis usually touches knee joints $[11,12]$. Latest research proves, that the risk of osteoarthrosis to appear in the knee joint of people with high BMI is two to ten times greater than in people with correct BMI. Also, in young people with higher BMI than usual, the risk of osteoarthrosis to appear is much greater, if it appears it happens much earlier, and the complaints start a lot sooner [11].

In every one of four patients with obesity the pain of knee joints can be observed. Published in the last years studies show, that losing the excess body mass lowers the risk of osteoarthrosis to appear by almost 2 times [11].

In obese people arthrosis appears not only in joints which are prone to mechanical loads but also in joints in hands which are not connected to such forces, but it is believed that the appearance of arthrosis in hand joints is connected to metabolic impact of obesity on arthrosis. The statement, that obesity metabolically impacts the appearance of osteoarthrosis is yet to be discussed and has been studied in the last years. The metabolic effect of obesity on the development of arthrosis may be associated with adipokines produced by adipose tissue, which contribute to the development of low-grade inflammation also associated with arthrosis [7].

\section{THE AIM}

The aim of the study is collect information on the importance of obesity and adipokine synthesized by adipose tissue, which can be a kind of specific biomarkers in the management of osteoarthritis.

\section{REVIEW AND DISCUSSION}

\section{PATHOPHYSIOLOGY OF OSTEOARTHRITIS IN OBESITY}

Obesity in many ways changes the appearance of osteoarthritis. Factors connected to obesity that cause osteoarthritis can be categorized as mechanical, biomechanical and metabolic [11].

To mechanical factors connected to obesity counts the increased load on joints. Available information in studies connect the impact of the mechanical factor (increased body mass) on correct functioning of the joints. It has been proven, that because of the mechanical factor there is an appearance of an inflammatory state of the joint which is showed by the appearance of mechanoreceptors on the surface of the cartilage tissue. This explains, why compressing the cartilage leads to increased expression of interleukin 8. Synovium is also prone to mechanical impact and subchondral layer. In the process of mechanical compression small damage is done to the synovium and its secondary hyperplasia and the increase in density of the subchondral layer. Hardening of the layer is connected to the presence of osteoblasts with changed phenotype, which are responsible for the inflammatory state appearance in this layer, and later in the entire joint [13].

To high body mass also causes disturbances in the function of joints impacting their biomechanical properties. It relates to negative impact of to high BMI not only on the articular surface, but also on the neighboring structures such as muscles, tendons and ligaments, which are also the main structures creating the joint, helping it perform its functions [11].

Obesity leads to inflammatory state of the entire organism to appear, which has a low intensity, impacting the whole organism, also the skeletal system. Prolonged inflammatory state disturbs the homeostasis of the entire organism at the same time impacting the metabolic chain happening in systems. However, the impact of elevated BMI isn't yet fully known. It is known however, that in patients with obesity osteoarthrosis develops not only in hip or knee joints, but also in joints of the hand. This specific localization of arthrosis can be a proof of the impact of obesity on the homeostasis of the organism which is changed because of the metabolic changes in system. Additionally, it is known, that white fat tissue synthesizes a lot of substances affecting the organism, so it is contributing in maintaining the metabolic and hormonal balance of the organism $[11,14]$.

\section{FAT TISSUE AND IT IS IMPACT ON THE ORGANISM}

Fat tissue is a kind of hormonal organ, which impacts the metabolism and immunological system of the organism. This tissue synthesizes a lot of bioactive proteins and peptides. It is responsible for secretion inflammatory mediators, which in literature are called adipokines $[10,14]$. Fat tissue also synthesizes inflammatory cytokines such as: 
tumor necrosis factor type alfa (TNF- $\alpha$ ), and interleukins and many more [14].

Adipokines are produced only by the fat tissue. In the last years many substances have been listed as adipokines. In the beginning it was thought, that substances accepted as adipokines impact only metabolic changes happening in the organism. This however was proven wrong, because after many studies it is known, that they also impact the immune system and maintain the homeostasis $[10,14]$.

Substances from this group not only partake in pathophysiology of metabolic diseases, but also rheumatologic diseases, lupus and inflammation of joints and bones. Their presence in blood plasma was also confirmed in case of cardiovascular syndrome [10].

The effect of fat tissue is dependent on its's contents, so on type of cells that it is made of. Fat tissue consists of fat cells (adipocytes), host-vascular cells and nonfat cells, which involve macrophages. Also depending on type of fat tissue it can consist of different kinds of adipocytes. In obese people fat tissue consists mainly of hypertrophic adipocytes. The more of those in the matrix of fat tissue the more adipokines are produced [10].

Also, fat tissue is located not only in abdominal wall, but also inside joints. Fat tissue in joints also impacts the development of osteoarthrosis but in different way. The impact of this tissue can be measured basing on activity of the infrapatellar fat pad inside the knee joint, which as the rest of the fat tissue in the organism synthesizes proinflammatory cytokines, adipokines and growth factors. Infrapatellar fat pad produces the P substance, which irritates the nociceptive fibers causing pain to appear $[15,17]$.

Such a wide range of impact on homeostasis, and the formation of arthrosis can lead to a belief, that adipokines which are synthesized by fat tissue can have a much bigger impact in prevention against arthrosis than BMI, because fat located inside joints can be created not only in patients with high BMI, but also in patients with correct BMI [15-17].

\section{ADIPOKINES}

Adipokines are kind of proinflammatory cytokines which are synthesized by fat tissue and then are released into bloodstream. They are responsible for the feeling of satiety, development of certain types of obesity, but also impact metabolic changes happening in the entire organism. Because of their impact they also affect the development of inflammatory diseases of joints and bones. Adipokines affecting the development of arthrosis involve leptin, adiponectin, visfatin, resistin, chemerin [13].

\section{CHARACTERISTIC OF CERTAIN ADIPOKINES}

\section{LEPTIN}

Leptin is a polypeptide built of 146 amino acids. It is coded by genes connected directly to obesity. It is synthesized mainly by white fat tissue [4]. It is released into the blood- stream in its inactive form. In blood plasma it is presents in its active form and it is discarded by kidneys. In patients with kidney diseases the content of leptin in blood plasma is elevated [18-20]. Also, in women during menopause the content of this polypeptide is up to 3 times greater in comparison to men [10].

Leptin's basic function is to control the need and the amount of energy used by controlling the hypothalamus. It is level in blood plasma is strictly connected to body mass, BMI and the mass of fat tissue in people suffering from osteoarthrosis. The content of Leptin in blood plas$\mathrm{ma}$ is also connected to one's daily routine, and its level is physiologically higher in women. It is also connected to proinflammatory cytokines in patients with developing arthrosis. High level of leptin has a negative effect on chondrocytes because it stimulates the function of MMPs (matrix metalloproteinases), which increase the degradation of cartilage tissue $[4,10]$.

In a study performed by Griffin et all (2009) on an animal model in mice, researchers turned off the leptin receptor and confirmed, that the lack of this polypeptide in the organism affects the buildup of severe obesity [21]. At the same time, they proved that it did not change the frequency of osteoarthrosis appearance. This suggests, that obesity alone does not impact the appearance of arthrosis, but other factors must be considered. In the same study, the researchers confirmed that leptin has a direct impact on osteoarthrosis appearance, but its concentration is not connected to obesity [21].

Concentration of leptin in the synovial fluid is much greater than its concentration in blood plasma. It may suggest, that its function is more direct than general, which possibly corresponds to its function, even in patients with low BMI with high volume of fat localized around the joints [4]

\section{ADIPONECTIN}

Adiponectin is a protein consisting of 244 amino acids and synthesized mainly by fat tissue. This protein is produced also by the placenta, epithelial cells, myocytes, osteoblasts, liver and hypophysis cells. In blood plasma adiponectin is present in three different forms, which have been divided into three groups basing on their molecular weight. Substance with the lowest molecular mass is trimer (LMWlow molecular weight). Second to the biggest is heksomere (MMW- medium molecular weight). The biggest form of them all is 12-18mere (HMW-high molecular weight) [10].

Content of this substance lowers with the increase in BMI which was confirmed in obese people, and its level grows with the decrease of BMI. It should also be noted that similarly to leptin the concentration of adiponectin is slightly higher in women than in men. One specific thing about adiponectin is, that its content in comparison to other substances from this group is lower in patients suffering from osteoarthritis [10].

The most basic function of adiponectin is their impact on lowering the level of glucose in blood, and the level of triglycerides because these proteins stimulate the loss of 
fat mass through increase fat acids oxidation processes in specific areas $[22,23]$.

In patients with arthrosis the content of adiponectin were nearly 100 times higher in blood serum than in joint fluid [4].

Studies performed on colonies of cartilage tissue show, that adiponectin impacts the homeostasis of these cells, because it stimulates the work of TIMP (metallopeptidase inhibitor) and suppresses the function of interleukin 1 beta (IL-1 $\beta$ ) and matrix metalloproteinases. Additionally, this substance impacts the expression of interleukin IL-10, which affects the macrophages and at the same time stimulates the function of TIMP-1 (metallopeptidase inhibitor 1), which protects the matrix against degradation [24].

In pathological states such as osteoarthritis, adiponectin also increases the synthesis of interleukin 6 (IL-6), interleukin 10 (IL-10) and matrix metalloproteinase 3 (MMP-3), which most likely is connected to lowered expression of TIMP. This affects the loss of activity of phagocytes and lymphocytes $\mathrm{T}[24,25]$.

\section{VISFATIN}

Visfatin is also called a factor increasing colonies of B cells and phosphoribosyl transferase. It impacts the insulin receptors because it functions as it is equivalent. Many factors may lead to the elevation of the level of this substance in the organism. In patients with obesity the level of visfatin is elevated, like patients with type II diabetes, polycystic ovary syndrome and articular inflammation. It should also be noted, that adipokines is produced in a higher volume by visceral fat tissue in comparison to subcutaneous fat tissue. This substance has a great impact on homeostasis of the entire organism, which is proven by studies, in which removal of this substance in experimental groups of animals lead to their death [26,27].

Studies conducted in the last years showed, that a high level of visfatin caused the degradation of cartilage tissue, which is connected to elevated expression of MMP-3 (matrix metalloproteinase 3) and MMP-13 (matrix metalloproteinase 13). This study was conducted on colonies of cartilage tissue, but there is however a lack of proof on negative effect of this substance on hyaline cartilage tissue in patients and correlation of this substance with the appearance of arthrosis [26, 27].

Studies conducted by Duan et all. In 2012 show, that in patients with primary osteoarthrosis the amount of visfatin in synovial fluid was much higher than in patients from control group. It should also be noted, that increase in concentration of this substance was positively correlated with expression of markers impacting the degradation of cartilage tissue [26].

\section{RESISTIN}

Resistin is a polypeptide with low molecular mass of $12,5 \mathrm{kDa}$ [28]. It is produced by fat tissue but also by the pancreas, bone marrow, lungs and inflammatory cells. It is content in blood plasma is positively correlated with fat mass. There however is no correlation of it is amounts in synovial fluid, because in patients with high BMI we can observe an increase of this substance in blood plasma, but it is amount in synovial fluid remains unchanged [4]. Elevated level of this adipokine can be observed in patients with inflammatory bowel disease, rheumatoid arthritis, but also in patients with asthma [29]. The mechanism of resistin have not been fully learned yet. Elevated level of this adipokine was observed in patients with articular injuries [4].

Studies conducted by Koskinen et all. in 2014 showed, that resistin is present in synovial fluid during degenerative changes. It was proved, that resistin is positively correlated with interleukin IL-6 (interleukin 6), MMP-1 (matrix metalloproteinase 1) and MMP-3, which means, that this substance impacts the appearance of inflammatory states of the cartilage and its degeneration [29].

\section{CHEMERIN}

Chemerin is a adipokine, that in the last years have been the object of many studies. Initially it was called chemokine [30]. It impacts the proinflammatory markers, affecting the level of reactive protein C and TNF-a (tumor necrosis factor alfa) in blood plasma. This adipokine affects the cartilage tissue. A high level of chemerin stimulates the inflammatory reaction in cartilage which is induced by chondrocytes [31].

Studies performed by Huang et all in 2012 on patients suffering from arthritis showed the lack of increase of chemerin in blood plasma in comparison to the control group [31]. This study however did not measure the level of this adipokine in the synovial fluid, and the experimental group was too small. Other studies, which checked the concentration of chemerin showed its presence in blood plasma in patients suffering from osteoarthritis. Its presence in blood plasma was positively correlated with the stage of the disease and the BMI. In comparison to other adipokines the level of chemerin in blood plasma is not dependent on gender or age, which makes it more useful as a biomarker and could ease additional analysis [10].

\section{CONCLUSION}

Osteoarthritis is a very serious condition, which touches more and more people. There are many factors affecting the appearance of the disease. The process of formation of the disease is not fully known yet. One of the reasons for arthrosis to appear is longer life expectancy and the increase of obesity. The disease is diagnosed only after symptoms appear. However, nowadays studies aim to check the relevance of biomarkers in presence of the disease, and possibly controlling the disease.

One of such biomarkers are adipokines, which are synthesized by fat tissue, which links them to the appearance of osteoarthritis because of BMI and body mass. However, the sole level of adipokines in synovial fluid is higher than 
in blood plasma, which shows, that their action is local, not global. It should also be noted, that some studies show the appearance of several adipokines in synovial fluid in patients suffering from osteoarthritis but with a normal BMI, which may suggest the activation of infrapatellar fat pad cells of the knee joint.

These substances may be a very useful way of examining the presence of osteoarthritis and may even be a way of controlling the disease. Further studies should be conducted to prove the hypothesis, that adipokines function as biomarkers for this disease.

\section{REFERENCES}

1. Michael JW, Schlüter-Brust KU, Eysel P. The epidemiology, etiology, diagnosis, and treatment of osteoarthritis of the knee. Dtsch Arztebl Int. 2010;107(9):152-162. doi: 10.3238/arztebl.2010.0152.

2. Klußmann A, Gebhardt $H$, Liebers F. Individual and occupational risk factors for knee osteoarthritis - Study protocol of a case control study. BMC Musculoskelet Disord. 2008;9(26):1-8. doi: 10.1186/1471-24749-26.

3. Hunter DJ, March L, Sambrook PN. Knee osteoarthritis: The influence of environmental factors. Clin Exp Rheumatol. 2002;(20):93-100.

4. PoonpetT, HonsawekS. Adipokines: Biomarkers for osteoarthritis. World J Orthop. 2014;5(3):319-327. doi: 10.5312/wjo.v5.i3.319.

5. Shekhar ACh, Kumar SA, Mritunjai S. Epidemiology of osteoarthritis and its association with ageing. IRJMST. 2015;6:21-39.

6. Chojnacki M, Kwapisz A, Synder M et al. Osteoarthritis: etiology, risk factors, molecular mechanisms. Post Hig (Online). 2014;2(68):640-652.

7. Palazzo C, Nguyen C, Lefevre-Colau MM et al. Risk factors and burden of osteoarthritis. Ann Phys Rehabil Med. 2016;59(3):134-138.

8. Altman RD. Early management of osteoarthritis. Am J Manag Care. 2010;16(Suppl):41-47.

9. Wang $X$, Hunter $D, X u J$ et al. Metabolic triggered inflammation in osteoarthritis. Osteoarthritis Cartilage. 2015;23:22-30. doi: 10.1016/j. joca.2014.10.002.

10. Azamar-Llamas D, Hernández-Molina G, Ramos-Ávalos B et al. Adipokines Contribution to the Pathogenesis of Osteoarthritis. Mediators Inflamm. 2017;5468023:1-26. doi: 10.1155/2017/5468023.

11. King LK, March L, Anandacoomarasamy A. Obesity and osteoarthritis. Indian J Med Res. 2013;138(2):185-193.

12. Jiang $L$, Rong J, Wang Y et al. The relationship between body mass index and hip osteoarthritis: a systematic review and meta-analysis. Joint Bone Spine. 2011;78:150-155. doi: 10.1016/j.jbspin.2010.04.011.

13. Berenbaum F, Eymard F, Houard X. Osteoarthritis, inflammation and obesity. Curr Opin Rheumatol. 2013;25(1):114-118. doi: 10.1097/ BOR.0b013e32835a9414.

14. Kluzek S, Newton JL, Arden NK. Is osteoarthritis a metabolic disorder? Br Med Bull. 2015;115(1):111-121. doi: 10.1093/bmb/ldv028.

15. Sudoł-Szopińska I, Kontny E, Zaniewicz-Kaniewska K et al. Role of inflammatory factors and adipose tissue in pathogenesis of rheumatoid arthritis and osteoarthritis. Part I: Rheumatoid adipose tissue. J Ultrason. 2013;13(53):192-201. doi: 10.15557/JoU.2013.0019.

16. Mazurek T.: Proinflammatory capacity of adipose tissue--a new insights in the pathophysiology of atherosclerosis. Kardiol Pol. 2009;67(10):1119-1124.

17. Wei W, Rudjito E, Fahy $N$ et al. The infrapatellar fat pad from diseased joints inhibits chondrogenesis of mesenchymal stem cells. Eur Cell Mater. 2015;2(30):303-314. doi: 10.22203/eCM.v030a21.
18. Scotece $M$, Mobasheri A. Leptin in osteoarthritis: Focus on articular cartilage and chondrocytes. Life Sci. 2015;1(140):75-78. doi: 10.1016/j. Ifs.2015.05.025.

19. Karvonen-Gutierrez CA, Harlow SD, Mancuso P et al. Association of leptin levels with radiographic knee osteoarthritis among a cohort of midlife women. Arthritis Care Res (Hoboken). 2013;65(6):936-944. doi: 10.1002/acr.21922.

20. Stannus OP, Cao Y, Antony B et al. Cross-sectional and longitudinal associations between circulating leptin and knee cartilage thickness in older adults. Ann Rheum Dis. 2015;74(1):82-88. doi: 10.1136/ annrheumdis-2013-203308.

21. Griffin TM, Huebner JL, Kraus VB et al. Extreme obesity due to impaired leptin signaling in mice does not cause knee osteoarthritis. Arthritis Rheum. 2009;60(10):2935-2944. doi: 10.1002/art.24854.

22. Naot D, Musson DS, Cornish J. The activity of adiponectin in bone. Calcif Tissue Int. 2017;100(5):486-499. doi: 10.1007/s00223-0160216-5.

23. Wu CL, Kimmerling KA, Little D et al. Serum and synovial fluid lipidomic profiles predict obesity-associated osteoarthritis, synovitis, and wound repair. Sci Repo. 2017;44315(4):1-11. doi: 10.1038/srep44315.

24. Wang K, Xu J, Cai J et al. Serum levels of interleukin-17 and adiponectin are associated with infrapatellar fat pad volume and signal intensity alteration in patients with knee osteoarthritis. Arthritis Res Ther. 2016;18(193):1-7. doi: 10.1186/s13075-016-1088-9.

25. Koskinen A, Juslin S, Nieminen R et al. Adiponectin associates with markers of cartilage degradation in osteoarthritis and induces production of proinflammatory and catabolic factors through mitogen-activated protein kinase pathways. Arthritis Res Ther. 2011;13(6):R184:1-11. doi: 10.1186/ar3512.

26. Duan Y, Hao D, Li M et al. Increased synovial fluid visfatin is positively linked to cartilage degradation biomarkers in osteoarthritis. Rheumatol Int. 2012;2(4):985-990. doi: 10.1007/ s00296-010-1731-8.

27. Franco-Trepat E, Guillán-Fresco M, Alonso-Pérez A et al. Visfatin Connection: Present and Future in Osteoarthritis and 0steoporosis. J Clin Med. 2019;8(8):1178:1-18. doi: 10.3390/jcm8081178.

28. Li XC, Tian F, Wang F. Clinical significance of resistin expression in osteoarthritis: a meta-analysis. Biomed Res Int. 2014;208016:1-11. doi: 10.1155/2014/208016.

29. Koskinen A, Vuolteenaho K, Moilanen T et al. Resistin as a factor in osteoarthritis: synovial fluid resistin concentrations correlate positively with interleukin 6 and matrix metalloproteinases MMP1 and MMP-3. Scandi J Rheumatol. 2014;43(3):249-253. doi: 10.3109/03009742.2013.853096.

30. Belluzzi E, Hamza El H, Granzotto $M$ et al. Systemic and local adipose tissue in knee osteoarthritis. J Cell Physiol. 2017;232(8):1971 \-1978. doi:10.1002/jcp.25716.

31. Huang K, Du G, Li L et al. Association of chemerin levels in synovial fluid with the severity of knee osteoarthritis. Biomarkers. 2012;17(1):16-20. doi: 10.3109/1354750X.2011.634028.

\section{ORCID and contributionship:}

Jaromir Jarecki - 0000-0002-6866-3850 A,B,D,F

Agnieszka Tomczyk-Warunek - 0000-0002-5007-7327 B,D,E

Mateusz Wicha - 0000-0002-8867-8005 ${ }^{D}$

Anna Horecka- 0000-0002-8694-8542 ${ }^{D}$

Rudolf Blicharski - 0000-0002-3538-8688 ${ }^{D}$

Maciej Dobrzyński-0000-0003-2368-1534 ${ }^{B, D}$ 


\title{
Conflict of interest
}

Authors declare no conflict of interest

\author{
CORRESPONDING AUTHOR \\ Agnieszka Tomczyk-Warunek \\ Chair and Department of Rehabilitation and Orthopaedics, \\ Medical University in Lublin, Lublin, Poland \\ 8 (SPSK Nr 4) Jaczewskiego str., 20-090 Lublin, Poland \\ tel: +48665828618 \\ e-mail:a.tomczykwarunek@gmail.com
}

Received: 06.07.2020

Accepted: 07.09.2020

A - Work concept and design, B - Data collection and analysis, C - Responsibility for statistical analysis,

D-Writing the article, $\mathbf{E}$-Critical review, $\mathbf{F}-$ Final approval of the article 